\title{
The Equilibrium States of the Free Boson Gas
}

\author{
J. T. Lewis \\ Dublin Institute for Advanced Studies, Dublin, Ireland \\ J. V. Pulè \\ Mathematics Department, Royal University of Malta, Malta
}

Received October 2, 1973

\begin{abstract}
The generating functional of the cyclic representation of the canonical commutation relations for the equilibrium state of the free Boson gas is calculated, using a method due to Kac, as the thermodynamic limit of the grand canonical generating functional. The relation to the work of Araki and Woods is discussed.
\end{abstract}

\section{§ 1. Introduction}

We compute the generating functional of the cyclic representation of the canonical commutation relations corresponding to the equilibrium state of the free Boson gas. We use a method suggested by Kac [1] in his rigorous derivation of the London-Placzek formula for the pair distribution function, to obtain the thermodynamic limit of the grand canonical ensemble. This method enables us to treat a range of boundary conditions and to display their effect on the generating functional.

In $\S 2$ we review the results about representations of the CCR which we shall require, and in $\S 3$ we describe the procedure for passing to the thermodynamic limit and state as Theorem 1 our main result which describes the limiting form of the generating functional. A special case of our result has been announced by Weiringa [2]. As a step towards the proof of Theorem 1 we need to establish the existence of condensation; the essential result is stated as Theorem 2 . In $\S 4$ we sketch some applications of these results before proving them in $\S 5$. We discuss the relation with the work of Araki and Woods [3] in $\S 6$ where we construct the cyclic representation of the CCRs corresponding to the generating functional described in Theorem 1, and discuss some of its properties.

We are deeply indebted to Professor M. Kac for showing us his derivation of the London-Placzek formula before publication. We are grateful to Dr. E. B. Davies for many stimulating discussions. 


\section{§ 2. Representations of the CCR}

Let $M$ be a complex pre-Hilbert space with inner product $\langle\cdot, \cdot\rangle$. A representation of the CCR over $M$ on a Hilbert space $\mathscr{H}$ is a map $h \mapsto W(h)$ of $M$ into the group $\mathscr{U}(\mathscr{H})$ of unitary operators on $\mathscr{H}$ satisfying

where

$$
W\left(h_{1}\right) W\left(h_{2}\right)=\omega\left(h_{1}, h_{2}\right) W\left(h_{1}+h_{2}\right)
$$

$$
\omega\left(h_{1}, h_{2}\right)=\exp \left\{\frac{i}{2} \operatorname{Im}\left\langle h_{1}, h_{2}\right\rangle\right\},
$$

and such that for each $h \in M$ the map $\lambda \mapsto W(\lambda h)$ of $\mathbb{R}$ into $\mathscr{U}(\mathscr{H})$ is strongly continuous. The continuity condition ensures, via Stone's theorem, the existence of self-adjoint operators $R(h)$ such that

$$
W(h)=\exp \{i R(h)\}
$$

and the map $h \mapsto R(h)$ is real-linear. Using the $R(h)$ we construct annihilation and creation operators

$$
\begin{aligned}
\psi(h) & =2^{-1 / 2}\{R(h)+i R(i h)\}, \\
\psi^{*}(h) & =2^{-1 / 2}\{R(h)-i R(i h)\} .
\end{aligned}
$$

To each cyclic representation $\{W, \mathscr{H}, \Omega\}$ of the CCR with cyclic vector $\Omega$ there corresponds a generating functional $\mu: M \rightarrow \mathbb{C}$ given by

$$
\mu(h)=\langle\Omega, W(h) \Omega\rangle .
$$

It has shown by Araki [12] and Segal [13] that a functional $\mu: M \rightarrow \mathbb{C}$ is the generating functional of a cyclic representation of the CCR if and only if it satisfies the following conditions:

(i) $\mu(0)=1$.

(ii) for each $h \in M$ the map $\lambda \mapsto \mu(\lambda h)$ is continuous.

(iii) for each finite set of complex numbers $c_{1}, \ldots, c_{n}$ and elements $h_{1}, \ldots, h_{n}$ of $M$

$$
\sum_{i} \sum_{j} \mu\left(h_{i}-h_{j}\right) \omega\left(h_{i}, h_{j}\right) c_{i} \bar{c}_{j} \geqq 0 .
$$

Let $\mathscr{A}$ be a positive quadratic form defined on a domain $\mathscr{2}(\mathscr{A})$ which is dense in a Hilbert-space $\mathscr{H}$. It follows from the above result that the functional

$$
\mu(h)=\mu_{F}(h) \exp \left\{-\frac{1}{2} \mathscr{A}\left(h_{1}, h_{2}\right)\right\}
$$

defined on $\mathscr{Q}(\mathscr{A})$ is the generating functional of a cyclic representation of the CCR, where

$$
\mu_{F}(h)=\exp \left\{-\frac{1}{4}\|h\|^{2}\right\}
$$


is the generating functional of the Fock-Cook representation $\left\{W_{F}, \mathscr{J}(\mathscr{H}), \Omega_{F}\right\}$. It is straightforward to verify that for such $\mu$ we have

$$
\begin{gathered}
\left\langle\psi^{*}\left(h_{1}\right) \psi\left(h_{2}\right)\right\rangle=\mathscr{A}\left(h_{2}, h_{1}\right), \\
\left\langle\psi^{*}\left(h_{1}\right) \psi^{*}\left(h_{2}\right) \psi\left(h_{3}\right) \psi\left(h_{4}\right)\right\rangle \\
=\mathscr{A}\left(h_{3}, h_{1}\right) \mathscr{A}\left(h_{4}, h_{2}\right)+\mathscr{A}\left(h_{4}, h_{1}\right) \mathscr{A}\left(h_{3}, h_{2}\right),
\end{gathered}
$$

where we write $\langle C\rangle$ for $\langle\Omega, C \Omega\rangle$ where $\Omega$ is the cyclic vector of the representation determined by $\mu$.

We have not assumed that the quadratic form $\mathscr{A}$ is closable, but when $\mathscr{A}$ is closed we can say more. In this case there exists a positive operator $A$ whose domain $\mathscr{D}(A)$ coincides with $\mathscr{2}(\mathscr{A})$ on which

Putting

we have

and

$$
\mathscr{A}\left(h_{1}, h_{2}\right)=\left\langle A h_{1}, A h_{2}\right\rangle .
$$

$$
T=\left(1+2 A^{2}\right)^{1 / 2}
$$

$$
\mathscr{D}(T)=\mathscr{D}(A)
$$

$$
\mu(h)=\exp \left\{-\frac{1}{4}\|T h\|^{2}\right\} .
$$

Chaiken [4] has considered generating functionals of this form following the construction introduced by Araki and Woods [3]. Let $\mathscr{M}$ be the closure of the range of $A$ and let $J$ be a conjugation of $\mathscr{H}$ which commutes with $A$. Then define $W(h)$ on $\mathscr{J}(\mathscr{H}) \otimes \mathscr{J}(\mathscr{M})$ by

$$
W(h)=W_{F}\left(\left(1+A^{2}\right)^{1 / 2} h\right) \otimes W_{F}(A J h)
$$

where $\mathscr{J}(\mathscr{H}), \mathscr{J}(\mathscr{M})$ are the Fock spaces over $\mathscr{H}$ and $\mathscr{M}$ respectively. Then $\langle\Omega, W(h) \Omega\rangle=\exp \left\{-\frac{1}{4}\|T h\|^{2}\right\}$ for $h \in \mathscr{D}(T)$, where $\Omega=\Omega_{F} \otimes \Omega_{F}$ is cyclic for $W$. This representation is a factor, it is irreducible if and only if $A=0$. It is unitarily equivalent to a direct sum of Fock-Cook representations if and only if $A$ is Hilbert-Schmidt.

A generating functional determines a state of the $C^{*}$-algebra of the CCR (see Manuceau [5]); those determined by generating functionals of the form (2.1) are quasi-free (Manuceau and Verbeure [6]) and invariant under the gauge transformations $h \mapsto e^{i \alpha} h$.

\section{§ 3. The Thermodynamic Limit of the Grand Canonical State}

Let $\Lambda^{1}$ be a bounded region in $\mathbb{R}^{3}$ having unit volume, and which is star-shaped with respect to some interior point which we choose as origin. We shall require the boundary $\partial \Lambda^{1}$ to satisfy a regularity condition (see $\S 5$ ). For each $L>0$ let $\Lambda^{L}$ be the region

$$
\Lambda^{L}=\left\{x \in \mathbb{R}^{3}: L^{-1} x \in \Lambda^{1}\right\}
$$


and put $\mathscr{H}_{L}=L^{2}\left(\Lambda^{L}\right)$. Then $\Lambda^{L} \subseteq \Lambda^{L^{\prime}}$ and $\mathscr{H}_{L} \subseteq \mathscr{H}_{L^{\prime}}$, whenever $L \leqq L^{\prime}$. Take $H_{L}$ to be the self-adjoint operator on $\mathscr{H}_{L}$ determined by $-\frac{1}{2} \bar{\Delta}$ in $\Lambda^{L}$ and the boundary condition $\frac{\partial \phi}{\partial n}+a \phi=0$ on $\partial \Lambda^{L}$ where $\frac{\partial}{\partial n}$ is the directional derivative in the direction of the outward normal $n$ to $\partial \Lambda^{L}$. Let $N_{L}$ be the number operator on the Fock space $\mathscr{J}\left(\mathscr{H}_{L}\right)$ and let $H_{L}^{F}$ be the operator on $\mathscr{J}\left(\mathscr{H}_{L}\right)$ induced by $H_{L}$ on $\mathscr{H}_{L}$. For each $L$ we consider the grand canonical density operator

$$
\sigma_{\beta, z}^{L}=\exp \left\{-\beta\left(H_{L}^{F}-\mu N_{L}\right)\right\} / \operatorname{trace}\left[\exp \left\{-\beta\left(H_{L}^{F}-\mu N_{L}\right)\right\}\right]
$$

on $\mathscr{J}\left(\mathscr{H}_{L}\right)$. Using standard results about Fock space we can show that the generating functional

$$
\mu_{\beta, z}^{L}(h)=\operatorname{trace}\left[\sigma_{\beta, z}^{L} W_{F}(h)\right]
$$

defined for $h \in \mathscr{H}_{L}$ is given by

where

$$
\mu_{\beta, z}^{L}(h)=\mu_{F}(h) \exp \left\{-\frac{1}{2} \mathscr{A}_{\beta, z}^{L}(h, h)\right\}
$$

$$
\mathscr{A}_{\beta, z}^{L}(h, h)=\left\langle h, z\left(e^{\beta H_{L}}-z\right)^{-1} h\right\rangle
$$

where $\beta=1 / k T$ is the inverse temperature and $z=e^{\beta \mu}$ is the fugacity. We wish to determine the limit as $L \rightarrow \infty$ of $\mathscr{A}_{\beta, z}^{L}$ when the mean density $\bar{Q}$ is held fixed.

Let $\left\{\phi_{k}^{L}\right\}$ be an orthonormal set of eigenvectors of $H_{L}$ corresponding to the eigenvalues $E_{k}^{L}$ which are assumed ordered so that $0 \leqq E_{1}^{L}<E_{2}^{L}$ $\leqq E_{3}^{L} \leqq \cdots-$; then $\left\{\phi_{k}^{L}\right\}$ is a basis for $\mathscr{H}_{L}$ and $\phi_{k}^{L}$ is given in terms of $\bar{\phi}_{k}=\bar{\phi}_{k}^{1}$ by

and

$$
\phi_{k}^{L}(x)=L^{-3 / 2} \phi_{k}\left(L^{-1} x\right),
$$

$$
E_{k}^{L}=L^{-2} E_{k} \text {. }
$$

The number operator $N_{L}$ is given by

$$
N_{L}=\sum_{k=1}^{\infty} \psi^{*}\left(\phi_{k}^{L}\right) \psi\left(\phi_{k}^{L}\right)
$$

and the number of particles in the $k^{\text {th }}$ level is given by the operator

$$
n_{k}^{L}=\psi^{*}\left(\phi_{k}^{L}\right) \psi\left(\phi_{k}^{L}\right)
$$

Using (2.2) and (3.1) we have

$$
\begin{aligned}
\left\langle n_{k}^{L}\right\rangle & =\operatorname{trace}\left(n_{k}^{L} \sigma_{\beta, z}^{L}\right) \\
& =\mathscr{A}_{\beta, z}^{L}\left(\phi_{k}^{L}, \phi_{k}^{L}\right)=\frac{z}{e^{\beta E_{k}^{L}}-z},
\end{aligned}
$$


and

$$
\left\langle N_{L}\right\rangle=\sum_{k=1}^{\infty} \frac{z}{e^{\beta E_{k}^{L}}-z} .
$$

We require $\left\langle N_{L}\right\rangle$ to have the value $\varrho L^{3}$ where $\varrho$ is the mean density. This determines $z$ as a function of $L$ through the constraint

$$
\bar{\varrho}=\frac{1}{L^{3}} \sum_{k=1}^{\infty} \frac{z(L)}{e^{\beta E_{k}^{L}-z(L)}} .
$$

Our objective is to find

$$
\mu_{\beta, \bar{\varrho}}(h)=\lim _{L \rightarrow \infty} \mu_{\beta, z(L)}^{L}(h)
$$

for $h$ in some dense subset of $L^{2}\left(\mathbb{R}^{3}\right)$. It is sufficient to determine

then

$$
\mathscr{A}_{\beta, \bar{\varrho}}(h, h)=\lim _{L \rightarrow \infty} \mathscr{A}_{\beta, z(L)}^{L}(h, h) \text {, }
$$

$$
\mu_{\beta, \bar{\varrho}}(h)=\mu_{F}(h) \exp \left\{-\frac{1}{2} \mathscr{A}_{\beta, \bar{\varrho}}(h, h)\right\} .
$$

We interpret $\mu_{\beta, \bar{\varrho}}(h)$ as the generating functional for a representation of the CCR for an infinite free Boson gas with mean density $\bar{\varrho}$. Let $g_{\alpha}$ be the function defined on the interval $[0,1]$ by

$$
g_{\alpha}(z)=\sum_{n=1}^{\infty} \frac{z^{n}}{n^{\alpha}}, \quad \alpha>1,
$$

and let $\varrho_{c}$ be the critical density defined by

$$
\varrho_{c}=(2 \pi \beta)^{-3 / 2} g_{3 / 2}(1) \text {. }
$$

Let $\mathscr{D}$ be the space of $C^{\infty}$ functions on $\mathbb{R}^{3}$ having compact support.

Theorem 1. The quadratic form $\mathscr{A}_{\beta, \varrho}$ is given on $\mathscr{D}$ by

$$
\begin{aligned}
\mathscr{A}_{\beta, \bar{\varrho}}(h, h) & =\left\langle h, f_{\zeta} h\right\rangle, \quad \bar{\varrho}<\varrho_{c}, \\
& =\left(\bar{\varrho}-\varrho_{c}\right)\left|\phi_{1}(0)\right|^{2}|\hat{h}(0)|^{2}+\left\langle h, f_{1} h\right\rangle, \quad \bar{\varrho} \geqq \varrho_{c},
\end{aligned}
$$

where $\zeta$ is the unique root of the equation

and

$$
\bar{\varrho}=(2 \pi \beta)^{-3 / 2} g_{3 / 2}(\zeta)
$$

with

$$
\left(f_{z} h\right)(x)=\int_{\mathbb{R}^{3}} f_{z}(\|x-y\|) h(y) d y
$$

$$
f_{z}(s)=(2 \pi \beta)^{-3 / 2} \sum_{n=1}^{\infty} e^{-s^{2} / 2 n \beta} \frac{z^{n}}{n^{3 / 2}} .
$$

A necessary preliminary to the proof of this theorem is the determination of the asymptotic behaviour of the solution $z(L)$ of the mean density 
Eq. (3.4) for large values of $L$, or of the solution $\zeta(L)$ of the transformed equation

where

$$
\bar{\varrho}=\frac{1}{L^{3}} \sum_{k=1}^{\infty} \frac{\zeta(L)}{e^{\beta \eta_{k}^{L}}-\zeta(L)}
$$

so that

$$
\zeta(L)=z(L) \exp \left(-\beta E_{1}^{L}\right), \quad \eta_{k}^{L}=E_{k}^{L}-E_{1}^{L},
$$

$$
0=\eta_{1}^{L}<\eta_{2}^{L} \leqq \eta_{3}^{L} \leqq \cdots .
$$

Since $\left\langle n_{k}^{L}\right\rangle$ must be positive and finite for all $k$ we require $0 \leqq \zeta(L)<1$. The properties of $\zeta(L)$ are given by the following:

Theorem 2. For $\bar{\varrho}<\varrho_{c}, \frac{1}{L^{3}} \frac{\zeta(L)}{1-\zeta(L)} \rightarrow 0$ and $\zeta(L) \rightarrow \zeta$ where $\zeta$ is the root of the equation

$$
\bar{\varrho}=(2 \pi \beta)^{-3 / 2} g_{3 / 2}(\zeta) .
$$

For $\bar{\varrho} \geqq \varrho_{c}, \frac{1}{L^{3}} \frac{\zeta(L)}{1-\zeta(L)} \rightarrow \bar{\varrho}-\varrho_{c}$ and $\zeta(L) \rightarrow 1$.

\section{§ 4. Applications}

(i) Macroscopic occupation of the ground state.

From Theorem 2 we see that $L^{-3}\left\langle n_{1}^{L}\right\rangle$, the density of particles in the ground state, satisfies

$$
\begin{aligned}
L^{-3}\left\langle n_{1}^{L}\right\rangle=\frac{L^{-3} \zeta(L)}{1-\zeta(L)} & \rightarrow \bar{\varrho}-\varrho_{c}, & & \bar{\varrho} \geqq \varrho_{c}, \\
& \rightarrow 0, & & \varrho<\varrho_{c},
\end{aligned}
$$

while $L^{-3}\left\langle n_{k}^{L}\right\rangle=\frac{L^{-3} \zeta(L)}{e^{\beta \eta_{k}^{L}}-\zeta(L)} \rightarrow 0$ for all values of $\bar{\varrho}$ when $k>1$ since $\eta_{k}^{L}=\left(E_{k}-E_{1}\right) / L^{2}$. Thus we have proved that condensation into the ground state occurs when the mean density $\bar{\varrho}$ exceeds $\varrho_{c}$.

(ii) Off-diagonal long-range order.

Applying (2.1) to the generating functional $\mu_{\beta, \bar{e}}$ we have $\left\langle\psi^{*}\left(h_{1}\right) \psi\left(h_{2}\right)\right\rangle$ $=\mathscr{A}_{\beta, \bar{\varrho}}\left(h_{2}, h_{1}\right)$. Let $\left\langle\psi^{*}(x) \psi(y)\right\rangle$ denote the kernel of the quadratic form $\left\langle\psi^{*}\left(h_{1}\right) \psi\left(h_{2}\right)\right\rangle$ so that

$$
\left\langle\psi^{*}\left(h_{1}\right) \psi\left(h_{2}\right)\right\rangle=\int_{\mathbb{R}^{3} \times \mathbb{R}^{3}} h_{1}(x)\left\langle\psi^{*}(x) \psi(y)\right\rangle \overline{h_{2}(y)} d x d y .
$$


Then using (3.5) we have

$$
\begin{aligned}
\left\langle\psi^{*}(x) \psi(y)\right\rangle & =f_{\zeta}(\|x-y\|), \quad \bar{\varrho}<\varrho_{c}, \\
& =\left(\varrho-\varrho_{c}\right)\left|\phi_{1}(0)\right|^{2}+f_{1}(\|x-y\|), \quad \bar{\varrho} \geqq \varrho_{c} .
\end{aligned}
$$

From the definition (3.7) of $f_{z}$ we see that as $\|x-y\| \rightarrow \infty$

$$
\begin{aligned}
\left\langle\psi^{*}(x) \psi(y)\right\rangle & \rightarrow 0, & & \bar{\varrho}<\varrho_{c}, \\
& \rightarrow\left(\bar{\varrho}-\varrho_{c}\right)\left|\phi_{1}(0)\right|^{2}, & & \bar{\varrho} \geqq \varrho_{c},
\end{aligned}
$$

which is the behaviour characterizing condensation, according to Penrose and Onsager [7]. However the limiting value of $\left\langle\psi^{*}(x) \psi(y)\right\rangle$ above the critical density depends on the boundary conditions through the value of the ground-state wave-function at the origin. In the circumstances described in this paper $\phi_{1}(0)$ is never zero but this happens in the case of the gas in a rotating container; then there is macroscopic occupation of the ground state but no off-diagonal long-range order.

(iii) The two-particle reduced density matrix

$\mathrm{Kac}$ [1] has given a rigorous proof of the London-Placzek formula for the pair distribution function. For this he requires the two-particle reduced density matrix.

This can be read off from (2.3) and (3.5):

$$
\begin{aligned}
\left\langle\psi^{*}\right. & \left.\left(x_{1}\right) \psi^{*}\left(x_{2}\right) \psi\left(y_{1}\right) \psi\left(y_{2}\right)\right\rangle \\
= & f_{\zeta}\left(\left\|x_{1}-y_{2}\right\|\right) f_{\zeta}\left(\left\|x_{2}-y_{1}\right\|\right)+f_{\zeta}\left(\left\|x_{1}-y_{1}\right\|\right) f_{\zeta}\left(\left\|x_{2}-y_{2}\right\|\right), \quad \bar{\varrho}<\varrho_{c}, \\
= & f_{1}\left(\left\|x_{1}-y_{2}\right\|\right) f_{1}\left(\left\|x_{2}-y_{1}\right\|\right)+f_{1}\left(\left\|x_{1}-y_{1}\right\|\right) f_{1}\left(\left\|x_{2}-y_{2}\right\|\right) \\
& +\left(\bar{\varrho}-\varrho_{c}\right)\left|\phi_{1}(0)\right|^{2}\left\{f_{1}\left(\left\|x_{1}-y_{2}\right\|\right)+f_{1}\left(\left\|x_{2}-y_{1}\right\|\right)+f_{1}\left(\left\|x_{1}-y_{1}\right\|\right)\right. \\
& \left.+f_{1}\left(\left\|x_{2}-y_{2}\right\|\right)\right\}+2\left(\varrho-\varrho_{c}\right)^{2}\left|\phi_{1}(0)\right|^{4}, \quad \bar{\varrho} \geqq \varrho_{c} .
\end{aligned}
$$

\section{§. Proof of the Theorems}

First we sketch a proof of the theorems and state the estimates which are required to clinch matters. The main idea is due to Kac [1]. The Green's function for the heat equation in $\Lambda^{1}$ is given by

$$
G(x, y ; t)=\sum_{k=1}^{\infty} \overline{\phi_{k}(x)} \phi_{k}(y) e^{-t E_{k}} .
$$

For small $t$ "the effect of the boundary is not felt" (see Kac [1]) and we expect

$$
G(x, y ; t) \sim(2 \pi t)^{-3 / 2} \exp \left\{-\|x-y\|^{2} / 2 t\right\}
$$


Putting $x=y$ and integrating over $\Lambda^{1}$ we get

$$
\sum_{k=1}^{\infty} e^{-t E_{k}} \sim(2 \pi t)^{-3 / 2}
$$

since the volume of $\Lambda^{1}$ is one. Using the explicit dependence of $E_{k}^{L}$ and $\phi_{k}^{L}$ on $L$ and interchanging the order of summation we have

where

$$
L^{-3} \sum_{k=1}^{\infty} \frac{\zeta(L)}{e^{\beta \eta_{k}^{L}}-\zeta(L)}=\sum_{n=1}^{\infty} \zeta(L)^{n} S_{L}(n \beta)
$$

$$
S_{L}(s)=L^{-3} \sum_{k=1}^{\infty} e^{-s \eta_{k}^{L}}=e^{s E_{1}^{L}} L^{-3} \sum_{k=1}^{\infty} e^{-s E_{k}^{L}} \sim(2 \pi s)^{-3 / 2}
$$

for large $L$, so that

$$
L^{-3} \sum_{k=1}^{\infty} \frac{\zeta(L)}{e^{\beta \eta_{k}^{L}}-\zeta(L)} \sim(2 \pi \beta)^{-3 / 2} g_{3 / 2}(\zeta)
$$

Now $g_{3 / 2}$ is continuous on $[0,1]$ and increases monotonically to a maximum $2.612 \ldots$ at $\zeta=1$. For $\bar{Q}<\varrho_{c}$ there is a unique $\zeta$ satisfying

$$
\bar{\varrho}=(2 \pi \beta)^{-3 / 2} g_{3 / 2}(\zeta) \text {. }
$$

For $\varrho \geqq \varrho_{c}$ we have to go back to the series (3.8). The first term dominates as $z(L)$ gets close to $e^{\beta E_{1}^{L}}$ and so we remove it and define

$$
S_{L}^{\prime}(s)=L^{-3} \sum_{k=2}^{\infty} e^{-s \eta_{k}^{L}}
$$

and prove

Lemma 1. Let $f_{L}(\zeta)=\sum_{n=1}^{\infty} \zeta^{n} S_{L}^{\prime}(n \beta)$. Then $f_{L}(\zeta) \rightarrow(2 \pi \beta)^{-3 / 2} g_{3 / 2}(\zeta)$ uniformly in $[0,1]$ so that if

then

$$
\lim _{L \rightarrow \infty} \zeta(L)=\zeta_{0} \in[0,1]
$$

$$
f_{L}(\zeta(L)) \rightarrow(2 \pi \beta)^{-3 / 2} g_{3 / 2}\left(\zeta_{0}\right) .
$$

Using this we are able to prove Theorem 2 .

To prove Theorem 1 we define

and

$$
T_{L}^{\prime}(s)=\sum_{k=2}^{\infty} e^{-s \eta_{k}^{L}}\left|\left\langle\phi_{k}^{L}, h\right\rangle\right|^{2}
$$

$$
T_{L}(s)=\left|\left\langle\phi_{1}^{L}, h\right\rangle\right|^{2}+T_{L}^{\prime}(s),
$$


for $h$ in the Schwartz space $\mathscr{D}$ and $L>L_{0}$ where $L_{0}$ is chosen so that the support of $h$ is contained in $\Lambda^{L_{0}}$. Putting

$$
K(s)=\int_{\mathbb{R}^{3} \times \mathbb{R}^{3}} \overline{h(x)} h(y) \exp \left\{-\|x-y\|^{2} / 2 s\right\} d x d y
$$

we use (5.1) to prove

Lemma 2. Suppose that $\lim _{L \rightarrow \infty} \zeta(L)=\zeta_{0} \in[0,1]$. Then

$$
\lim _{L \rightarrow \infty} \sum_{n=1}^{\infty} \zeta(L)^{n} T_{L}^{\prime}(n \beta)=(2 \pi \beta)^{-3 / 2} \sum_{n=1}^{\infty} \zeta_{0}^{n} K(n \beta) .
$$

In proving the lemmas we need the following estimates. Let $G_{c}(x, y ; t)$ $=G(x, y ; t)-(2 \pi t)^{-3 / 2} \exp \left\{-\|x-y\|^{2} / 2 t\right\}$ where $G(x, y ; t)$ is the Green's function for the heat equation

$$
\frac{\partial \psi}{\partial t}=-\frac{1}{2} \Delta \psi \quad \text { in } \quad \Lambda^{1}
$$

subject to the boundary condition

$$
\frac{\partial \psi}{\partial n}+\alpha \psi=0 \quad \text { on } \quad \partial \Lambda^{1}
$$

Then, provided the boundary is sufficiently regular, for each $T<\infty$ there exists a constant $c(T)$ such that

$$
\left|G_{c}(x, y ; t)\right| \leqq \frac{c(T)}{t^{3 / 2}} \exp \left\{-l_{y}^{2} / 2 t\right\}
$$

for all $t<T$, where $l_{y}$ is the minimum distance of $y$ from $\partial \Lambda^{1}$. Furthermore there exist constants $a$ and $b$ such that for $k$ sufficiently large

and for all

$$
\begin{gathered}
E_{k}>b k^{1 / 2} \\
x \in \Lambda^{1},\left|\phi_{k}(x)\right| \leqq a E_{k} .
\end{gathered}
$$

These can be extracted from Arima [8] and Mizohata and Arima [9]. See also Pulè [11].

We first prove the Lemmas. We prove the harder of the two, Lemma 2, and indicate how the proof can be modified to prove Lemma 1.

Proof of Lemma 2. We first prove that for each $T<\infty$ there exists a constant $c(T)$ such that for all $n<T L^{2} / \beta$ we have

$$
\left|T_{L}(n \beta)-K(n \beta)\right|<L^{-1 / 2} n^{-5 / 4} c(T) .
$$

Using the estimates $(5.4,5)$ we have for $k$ sufficiently large

$$
\left|e^{-t E_{k}^{L}} \bar{\phi}_{k}^{L}(x) \phi_{k}^{L}(y)\right| \leqq A e^{-t E_{k}^{L}}\left(E_{k}^{L}\right)^{2} \leqq A \frac{6 !}{t^{6}} \frac{\left(E_{k}^{L}\right)^{2}}{\left(E_{k}^{L}\right)^{6}} \leqq B t^{-6} k^{-2},
$$


so that

$$
\sum_{k=1}^{\infty} e^{-t E_{k}^{L}} \bar{\phi}_{k}^{L}(x) \phi_{k}^{L}(y)=G^{L}(x, y ; t)
$$

is absolutely and uniformly convergent for $x, y$ in $\Lambda^{L}$. Define $K: \mathscr{H}^{L} \rightarrow \mathscr{H}^{L}$ by

Then

$$
(K g)(x)=\int_{\Lambda^{L}} G^{L}(x, y ; t) g(y) d y .
$$

$$
\left(K \phi_{n}^{L}\right)(x)=e^{-t E_{n}^{L}} \phi_{n}^{L}(x)
$$

since the uniform convergence allows us to integrate term by term, and so $G^{L}$ is the Green's function of the heat Eq. (5.2).

Using the estimate (4.3), for $G_{c}^{L}$ defined by

we have

$$
G_{c}^{L}(x, y ; t)=G^{L}(x, y ; t)-(2 \pi t)^{-3 / 2} e^{-\|x-y\|^{2} / 2 t}
$$

where

$$
\left|G_{c}^{L}(x, y ; n \beta)\right|=\frac{1}{L^{3}}\left|G_{c}^{1}\left(\frac{x}{L}, \frac{y}{L} ; \frac{n \beta}{L^{2}}\right)\right| \leqq \frac{c(T)}{(n \beta)^{3 / 2}} e^{-\delta L^{2} / 2 n \beta}
$$

$$
\delta=\inf _{y \in \Lambda^{L_{0}}} d\left(y / L, \partial \Lambda^{\prime}\right)=\inf _{y \in \Lambda^{L_{0} / L}} d\left(y, \partial \Lambda^{\prime}\right)=d\left(\Lambda^{L_{0} / L}, \partial \Lambda^{\prime}\right) .
$$

Choosing $L \geqq 2 L_{0}, \delta \geqq d\left(\Lambda^{1 / 2}, \partial \Lambda^{1}\right)>0$, so that

$$
\left|G_{c}^{L}(x, y ; n \beta)\right|<\frac{c(T)}{(n \beta)^{3 / 2}}\left(\frac{n \beta}{2 \delta L^{2}}\right)^{1 / 4}=c^{\prime}(T) n^{-5 / 4} L^{-1 / 2} \text {. }
$$

Using uniformity of convergence in $x$ and $y$ of (4.9) we have

$$
\begin{aligned}
T_{L}(n \beta) & =e^{n \beta E_{1} / L^{2}} \sum_{k=1}^{\infty} e^{-n \beta E_{k}^{L}}\left|\left\langle\phi_{k}^{L}, h\right\rangle\right|^{2} \\
& =e^{n \beta E_{1} / L^{2}} K(n \beta)+e^{n \beta E_{1} / L^{2}} \int \overline{h(x)} h(y) G_{c}^{L}(x, y ; n \beta) d x d y
\end{aligned}
$$

so that

$$
\left|T_{L}(n \beta)-K(n \beta)\right| \leqq|K(n \beta)|\left(e^{n \beta E_{1} / L^{2}}-1\right)+n^{-5 / 4} L^{-1 / 2} e^{T E_{1}} c^{\prime}(T) .
$$

For $n \beta L^{-2} \leqq T$ we have $e^{n \beta E_{1} / L^{2}}-1 \leqq\left(\frac{n \beta E_{1}}{L^{2}}\right)^{1 / 4} \frac{e^{T E_{1}}-1}{T^{1 / 4}}$ and

$$
|K(n \beta)| \leqq(2 \pi \beta n)^{-3 / 2}|\hat{h}(0)|^{2},
$$

and hence

$$
\left|T_{L}(n \beta)-K(n \beta)\right| \leqq n^{-5 / 4} L^{-1 / 2} c^{\prime \prime}(T)
$$


To prove the Lemma it is enough to prove that given $\varepsilon>0$ there exists $L^{\prime}$ such that for all $L>L^{\prime}$

$$
\left|\sum_{n=1}^{\infty} \zeta^{n} T_{L}^{\prime}(n \beta)-\sum_{n=1}^{\infty} \zeta^{n} K(n \beta)\right|<\varepsilon
$$

since by uniform convergence $\sum_{n=1}^{\infty} \zeta^{n} K(n \beta)$ is continuous on $[0,1]$. Since, as a function of $n, n^{5 / 4} \exp \left(-\beta n E / L^{2}\right)$ is monotonic decreasing for $n \beta E L^{-2}>5 / 4$ we have $n^{5 / 4} T_{L}^{\prime}(n \beta)$ is non-increasing for $\beta n \eta_{2} L^{-2}>5 / 4$. Put $T=5 / 4 \eta_{2}^{-1}+1$ in (5.6), and let $N=N(L)$ be the largest integer not greater than $(T-1) L^{2} / \beta$, then for $n<N(L)+1$ the results (5.6) holds and

$$
N(L)+1>(T-1) L^{2} / \beta=\frac{5 L^{2}}{4 \beta \eta_{2}}
$$

so that $n^{5 / 4} T_{L}^{\prime}(n \beta)$ is non-increasing for all $n>N(L)$. Now

$$
\begin{aligned}
& \left|\sum_{n=1}^{\infty} \zeta^{n} T_{L}^{\prime}(n \beta)-\sum_{n=1}^{\infty} \zeta^{n} K(n \beta)\right| \leqq \mathrm{I}+\mathrm{II}+\mathrm{III} \\
& \quad=\sum_{n=N(L)+1}^{\infty} T_{L}^{\prime}(n \beta)+\sum_{n=N(L)+1}^{\infty} K(n \beta)+\sum_{n=1}^{N(L)}\left|T_{L}^{\prime}(n \beta)-K(n \beta)\right| .
\end{aligned}
$$

But $\phi_{1}(x)$ is continuous at $x=0$, being an eigenfunction of an elliptic operator, so that

$L^{3 / 2}\left\langle\phi_{1}^{L}, h\right\rangle=\int_{\Lambda^{L}} \overline{\phi_{1}\left(\frac{x}{L}\right)} h(x) d x \rightarrow \overline{\phi_{1}(0)} \int_{\mathbb{R}^{3}} h(x) d x=\overline{\phi_{1}(0)} \hat{h}(0)$.

Thus there is a constant $D(h)$ such that $\left|\left\langle\phi_{1}^{L}, h\right\rangle\right|^{2}<D(h) L^{-3}$ for $L$ sufficiently large to that

$$
\begin{aligned}
\mathrm{III} & \leqq \sum_{n=1}^{N}\left|T_{L}(n \beta)-K(n \beta)\right|+N D(h) L^{-3} \\
& <\frac{c^{\prime \prime}(T)}{L^{1 / 2}} \sum_{n=1}^{N} n^{-5 / 4}+\frac{(T-1) D(h)}{\beta L}<\frac{c^{\prime \prime}(T)}{L^{1 / 2}} g_{5 / 4}(1)+\frac{(T-1) D(h)}{\beta L} .
\end{aligned}
$$

Now

$$
\begin{aligned}
\mathrm{I}< & (N+1)^{5 / 4} T_{L}^{\prime}(\beta(N+1)) \sum_{n=N+1}^{\infty} n^{-5 / 4}<(N+1)^{5 / 4} T_{L}^{\prime}(\beta(N+1)) g_{5 / 4}(1) \\
\leqq & (N+1)^{5 / 4} g_{5 / 4}(1)\left|T_{L}(\beta(N+1))-K(\beta(N+1))\right| \\
& +(N+1)^{5 / 4} g_{5 / 4}(1) K(\beta(N+1)) \\
< & L^{-1 / 2} c^{\prime \prime}(T) g_{5 / 4}(1)+(N+1)^{-1 / 4}|\hat{h}(0)|^{2} g_{5 / 4}(1)(2 \pi \beta)^{-3 / 2} \\
< & L^{-1 / 2} c^{\prime \prime}(T) g_{5 / 4}(1)+L^{-1 / 2}(T-1)^{-1 / 4} \beta^{1 / 4}|\hat{h}(0)|^{2} g_{5 / 4}(1)(2 \pi \beta)^{-3 / 2}
\end{aligned}
$$


Similarly II can be made arbitrarily small by choosing $L$ sufficiently large, and the proof of Lemma 2 is complete.

The proof of Lemma 1 is similar, with the estimate (5.6) replaced by

$$
\left|S_{L}(n \beta)-(2 \pi n \beta)^{-3 / 2}\right| \leqq n^{-5 / 4} L^{-1 / 2} c_{2}(T) \text { for } n<T L^{2} / \beta,
$$

which is proved as follows:

From (5.3) we have that for each $T<\infty$ there exists $c(T)<\infty$ such that

$$
\begin{aligned}
\int_{\Lambda^{1}}\left|G_{c}^{1}(x, x ; t)\right| d x & <\frac{c(T)}{t^{3 / 2}} \int_{\Lambda^{1}} e^{-l_{x}^{2} / 2 t} d x \\
& \leqq \frac{c^{\prime}(T)}{t^{3 / 2}} \int_{0}^{x} e^{-l_{x}^{2} / 2 t} d l_{x} \text { for some } X<\infty \\
& \leqq \frac{c^{\prime}(T)}{t^{5 / 4}} \int_{0}^{x} l_{x}^{-1 / 2} d l_{x} \leqq \frac{c_{1}(T)}{t^{5 / 4}}
\end{aligned}
$$

Using the uniformity of the convergence of (5.7) and (5.8) we have

$$
\begin{aligned}
S_{L}(n \beta) & =L^{-3} e^{n \beta E_{1} / L^{2}} \sum_{k=1}^{\infty} e^{-n \beta E_{k}^{L}}=L^{-3} e^{n \beta E_{1} / L^{2}} \int_{\Lambda^{L}} G^{L}(x, x ; n \beta) d x \\
& =(2 \pi n \beta)^{-3 / 2} e^{n \beta E_{1} / L^{2}}+L^{-3} e^{n \beta E_{1} / L^{2}} \int_{\Lambda^{L}} G_{c}^{L}(x, x ; n \beta) d x .
\end{aligned}
$$

Thus

$$
\begin{aligned}
\left|S_{L}(n \beta)-(2 \pi n \beta)^{-3 / 2}\right| \leqq & (2 \pi n \beta)^{-3 / 2}\left(e^{n \beta E_{1} / L^{2}}-1\right) \\
& +L^{-3} e^{n \beta E_{1} / L^{2}} \int_{\Lambda^{L}}\left|G_{c}^{L}(x, x ; n \beta)\right| d x \\
\leqq & L^{-1 / 2}(n \beta)^{-5 / 4} T^{-1 / 4} E_{1}^{1 / 4}\left(e^{T E_{1}}-1\right) \\
& +L^{-3} e^{T E_{1}} \int_{A^{1}}\left|G_{c}^{1}\left(x, x ; \frac{n \beta}{L^{2}}\right)\right| d x \\
\leqq & L^{-1 / 2} n^{-5 / 4} c_{2}(T) .
\end{aligned}
$$

Proof of Theorem 2. For $\zeta(L) \in[0,1)$

$$
\sum_{k=2}^{\infty} \frac{\zeta(L)}{e^{\beta \eta_{k}^{L}}-\zeta(L)}=\sum_{k=2}^{\infty} \sum_{n=1}^{\infty} \zeta(L) e^{-n \beta \eta_{k}^{L}} .
$$

The terms on the right hand side are positive and the series on the left hand side converges because of the estimate (5.4), so we may interchange the order of summation and get

$$
\sum_{k=2}^{\infty} \frac{\zeta(L)}{e^{\beta \eta_{k}^{L}}-\zeta(L)}=\sum_{n=1}^{\infty} \zeta(L)^{n} S_{L}^{\prime}(n \beta)=f_{L}(\zeta(L)),
$$


and we have

$$
\frac{L^{-3} \zeta(L)}{1-\zeta(L)}+f_{L}(\zeta(L))=\bar{\varrho}
$$

which implies that $f_{L}(\zeta(L))<\bar{\varrho}$.

Now consider case (a): $\varrho<\varrho_{c}$. Then there exists an $\varepsilon>0$ such that $\bar{\varrho}+\varepsilon<\varrho_{c}$ and by Lemma 1 there is an $L_{0}$ such that for all $L>L_{0}$

$$
(2 \pi \beta)^{-3 / 2} g_{3 / 2}(\zeta(L))<f_{L}(\zeta(L))+\varepsilon<\bar{\varrho}+\varepsilon<\varrho_{c} .
$$

Let $\zeta_{\varepsilon}$ be the unique root of $(2 \pi \beta)^{-3 / 2} g_{3 / 2}\left(\zeta_{\varepsilon}\right)=\bar{\varrho}+\varepsilon$. Then since $g_{3 / 2}$ is monotonic increasing

and

$$
\zeta(L)<\zeta_{\varepsilon}<1
$$

$$
\frac{\zeta(L)}{1-\zeta(L)}<K=\frac{\zeta_{\varepsilon}}{1-\zeta_{\varepsilon}} \text {. }
$$

Then $L^{-3} \zeta(L)(1-\zeta(L))^{-1}<L^{-3} K \rightarrow 0$ as $L \rightarrow \infty$. We wish to prove that $\lim _{L \rightarrow \infty} \zeta(L)=\zeta \quad$ where $\bar{\varrho}=(2 \pi \beta)^{-3 / 2} g_{3 / 2}(\zeta)$. But $\left|g_{3 / 2}(\zeta(L))-g_{3 / 2}(\zeta)\right|$ $=\left|g_{3 / 2}^{\prime}(\theta)\right||\zeta(L)-\zeta|$ for some $\theta \in[0,1)$ and $g_{3 / 2}^{\prime}(\theta) \geqq 1$ so that

$$
\begin{aligned}
|\zeta(L)-\zeta| & \leqq(2 \pi \beta)^{3 / 2}\left|(2 \pi \beta)^{-3 / 2} g_{3 / 2}(\zeta(L))-\bar{\varrho}\right| \\
& \leqq(2 \pi \beta)^{3 / 2}\left\{\left|f_{L}(\zeta(L))-\bar{\varrho}\right|+\mid f_{L}(\zeta(L))-\left(2 \pi \beta^{-3 / 2} g_{3 / 2}(\zeta(L)) \mid\right\}\right. \\
& \leqq(2 \pi \beta)^{3 / 2}\left(L^{-3} K+\varepsilon\right) .
\end{aligned}
$$

Case (b): $\bar{Q} \geqq \varrho_{c}$.

Given $\varepsilon>0$ choose $\delta$ such that

$$
2 \delta<\varrho_{c}-(2 \pi \beta)^{-3 / 2} g_{3 / 2}(1-\varepsilon) .
$$

Then there exists an $L_{0}$ such that for all $L>L_{0}$

and

$$
\frac{L^{-3}(1-\varepsilon)}{1-(1-\varepsilon)}<\delta
$$

$$
f_{L}(1-\varepsilon)<(2 \pi \beta)^{-3 / 2} g_{3 / 2}(1-\varepsilon)+\delta \text {, }
$$

so that

$$
\frac{L^{-3}(1-\varepsilon)}{1-(1-\varepsilon)}+f_{L}(1-\varepsilon)<(2 \pi \beta)^{-3 / 2} g_{3 / 2}(1-\varepsilon)+2 \delta<\varrho_{c} \leqq \bar{\varrho} .
$$

Thus

$$
\frac{L^{-3}(1-\varepsilon)}{1-(1-\varepsilon)}+f_{L}(1-\varepsilon)<\frac{L^{-3} \zeta(L)}{1-\zeta(L)}+f_{L}(\zeta(L))
$$


But $z \mapsto \frac{L^{-3} z}{1-z}+f_{L}(z)$ is monotonic increasing, so that

and so

$$
1>\zeta(L)>1-\varepsilon \text { for all } L>L_{0} \text {, }
$$

But

$$
\zeta(L) \rightarrow 1 \text {. }
$$

$$
\frac{L^{-3} \zeta(L)}{1-\zeta(L)}=\bar{\varrho}-f_{L}(\zeta(L))
$$

so by Lemma 2

$$
\frac{L^{-3} \zeta(L)}{1-\zeta(L)} \rightarrow \bar{\varrho}-\varrho_{c} .
$$

Proof of Theorem 1. We can write

$$
\begin{aligned}
\mathscr{A}_{\beta, z}^{L}(h, h) & =\left\langle h, z\left(e^{\beta H_{L}}-z\right)^{-1} h\right\rangle=\sum_{n=1}^{\infty} z^{n} \sum_{k=1}^{\infty} e^{-\beta n E_{k}^{L}}\left|\left\langle\phi_{k}^{L}, h\right\rangle\right|^{2} \\
& =\sum_{n=1}^{\infty} \zeta^{n} \sum_{k=1}^{\infty} e^{-\beta n \eta_{k}^{L}}\left|\left\langle\phi_{k}^{L}, h\right\rangle\right|^{2}=\sum_{n=1}^{\infty} \zeta^{n} T_{L}(n \beta) \\
& =\frac{\zeta}{1-\zeta}\left|\left\langle\phi_{1}^{L}, h\right\rangle\right|^{2}+\sum_{n=1}^{\infty} \zeta^{n} T_{L}^{\prime}(n \beta) \\
& =\frac{L^{-3} \zeta}{1-\zeta}\left|L^{3 / 2}\left\langle\phi_{1}^{L}, h\right\rangle\right|^{2}+\sum_{n=1}^{\infty} \zeta^{n} T_{L}^{\prime}(n \beta) .
\end{aligned}
$$

Using Lemma 2 and (5.9) we have

$$
\begin{aligned}
\mathscr{A}_{\beta, \bar{\varrho}}(h, h) & =\lim _{L \rightarrow \infty} \mathscr{A}_{\beta, z(L)}^{L}(h, h) \\
& =\left(\bar{\varrho}-\varrho_{c}\right)\left|\phi_{1}(0)\right|^{2}|\hat{h}(0)|^{2}+\sum_{n=1}^{\infty} K(n \beta), \quad \bar{\varrho} \geqq \varrho_{c}, \\
& =\sum_{n=1}^{\infty} \zeta^{n} K(n \beta), \quad \bar{\varrho}<\varrho_{c} .
\end{aligned}
$$

The series

$$
f_{z}(\|x-y\|)=(2 \pi \beta)^{-3 / 2} \sum_{n=1}^{\infty} n^{-3 / 2} z^{n} \exp \left\{-\|x-y\|^{2} / 2 n \beta\right\}
$$

converges uniformly in $x$ and $y$ and so we can put

$$
\sum_{n=1}^{\infty} z^{n} K(n \beta)=\left\langle h, f_{z} h\right\rangle \text {. }
$$

This completes the proof of Theorem 1 . 


\section{§ 6. Construction of the Representations}

It can be shown that

where

$$
\widehat{f_{z} h}(k)=\tilde{\varrho}_{z}(k) \hat{h}(k)
$$

$$
\tilde{\varrho}_{z}(k)=z\left(e^{\beta\|k\|^{2} / 2}-z\right)^{-1}
$$

so that (3.5) can be re-written

$$
\begin{aligned}
\mathscr{A}_{\beta, \bar{\varrho}}(h, h) & =\left(\bar{\varrho}-\varrho_{c}\right)\left|\phi_{1}(0)\right|^{2}|\hat{h}(0)|^{2}+\left\langle\hat{h}, \tilde{\varrho}_{1} \hat{h}\right\rangle, & & \bar{\varrho} \geqq \varrho_{c}, \\
& =\left\langle\hat{h}, \tilde{\varrho}_{z} \hat{h}\right\rangle, & & \bar{\varrho}<\varrho_{c} .
\end{aligned}
$$

where $z$ satisfies

$$
\bar{\varrho}=\int_{\mathbb{R}^{3}} \tilde{\varrho}_{z}(k) d k .
$$

It is clear that our result agrees with that obtained by Araki and Woods [3] for $\bar{\varrho}<\varrho_{c}$ but for $\bar{\varrho} \geqq \varrho_{c}$ they have

where

$$
\mu_{\beta, \varrho}^{A}(h)=\mu_{F}(h) \exp \left\{-\frac{1}{2}\left\langle\hat{h}, \varrho_{1} \hat{h}\right\rangle\right\} J_{0}\left(\left(2 \varrho_{0}\right)^{1 / 2}|\hat{h}(0)|\right)
$$

$$
\varrho_{0}=\bar{\varrho}-\varrho_{c} .
$$

The relation between the two can be seen by putting $\phi_{1}(0)=1$ (corresponding to the boundary condition $\frac{\partial \phi}{\partial n}=0$ ) and using the identity

where

$$
\begin{aligned}
\exp \left(-\frac{1}{2} s|x|^{2}\right) & =\int_{0}^{\infty} J_{0}\left((2 t)^{1 / 2}|x|\right) e^{-t / s} \frac{d t}{s} \\
\mu_{\beta, \bar{\varrho}}(h) & =\int_{0}^{\infty} \mu_{\beta, \varrho}^{A W}(h) K(\varrho ; \bar{\varrho}) d \varrho
\end{aligned}
$$

$$
\begin{aligned}
K(\varrho ; \bar{\varrho}) & =\left(\bar{\varrho}-\varrho_{c}\right)^{-1} \exp \left\{-\left(\varrho-\varrho_{c}\right) /\left(\bar{\varrho}-\varrho_{c}\right)\right\}, & & \varrho>\varrho_{c}, \\
& =0, & & \varrho \leqq \varrho_{c} .
\end{aligned}
$$

But this is just the expression discovered by Kac [1] for the probability distribution of the density in the thermodynamic limit of the grand canonical ensemble corresponding to mean density $\bar{\varrho}$; so that, by fixing the density in the ground state, Araki and Woods obtained the correct generating functional given that the density in the ground state is $\varrho_{0}$. Since the first draft of this paper was written Cannon [14] has proved that the Araki-Woods state is the thermodynamic limit of the canonical state. In discussing the grand canonical generating functional we shall concentrate on the case $\bar{\varrho} \geqq \varrho_{c}$ since for $\bar{\varrho}<\varrho_{c}$ it is the same as the canonical which has been treated exhaustively (see Araki and Woods [3] and Hugenholtz [10]). 
The onset of off-diagonal long range order corresponds to the quadratic form $\mathscr{A}_{\beta, \bar{Q}}$ becoming non-closable, because of the term $\left(\bar{\varrho}-\varrho_{c}\right)\left|\phi_{1}(0)\right|^{2}|\hat{h}(0)|^{2}$. For this reason the generating functional $\mu_{\beta, \bar{\varrho}}$ is not of the form

$$
\exp \left\{-\frac{1}{4}\|T h\|^{2}\right\}
$$

and in fact is not a factor, and we cannot use Chaiken's results [4]. To obtain a representation having $\mu_{\beta, \bar{\varrho}}$ as generating functional with $\bar{\varrho}>\varrho_{c}$, let $\mathscr{H}_{0}$ be $L^{2}\left(\mathbb{R}^{2}, K(r ; \varrho) d r d \theta / 2 \pi\right)$. Define $W_{0}(z)$ on $\mathscr{H}_{0}$ for all $z=p+i q \in \mathbb{C}$ by

$$
W_{0}(p+i q) g(r, \theta)=\exp \left\{i 2^{1 / 2}\left(r-\varrho_{c}\right)^{1 / 2}(p \cos \theta+q \sin \theta)\right\} g(r, \theta) .
$$

Let $\Omega_{0}$ be the element of $\mathscr{H}_{0}$ which is identically equal to 1 , then $\left\{W_{0}, \mathscr{H}_{0}, \Omega_{0}\right\}$ is a cyclic representation of the abelian group $\mathbb{C}$ with generating functional

$$
\begin{aligned}
\mu_{0}(z) & =\int_{0}^{\infty} \int_{0}^{2 \pi} \exp \left\{i 2^{1 / 2}\left(r-\varrho_{c}\right)^{1 / 2}(p \cos \theta+q \sin \theta)\right\} K(r ; \bar{\varrho}) d r d \theta / 2 \pi \\
& =\int_{0}^{\infty} J_{0}\left(2^{1 / 2}\left(r-\varrho_{c}\right)^{1 / 2}|z|\right) K(r ; \bar{\varrho}) d r \\
& =\exp \left\{-\frac{1}{2}\left(\bar{\varrho}-\varrho_{c}\right)|z|^{2}\right\} .
\end{aligned}
$$

We have obtained $\mu_{\beta, \bar{\varrho}}(h)$ for $h$ in the Schwartz space $\mathscr{D}$ but it can be extended to larger spaces of test-functions and it is convenient to use the maximal extension which is to the domain $\mathscr{2}\left(\mathscr{A}_{\beta, \bar{\rho}}\right)$ of the quadratic form. This is the intersection of the domains of the singular part $|\hat{h}(0)|^{2}$ and the closed part $\left\langle h, f_{1} h\right\rangle$. The domain of the singular part is $L^{1}\left(\mathbb{R}^{3}\right)$ $\cap L^{2}\left(\mathbb{R}^{3}\right)$ and this is contained in the domain of the closed part since for $h \in L^{1}\left(\mathbb{R}^{3}\right) \cap L^{2}\left(\mathbb{R}^{3}\right)$

$$
\int_{\mathbb{R}^{3}} \tilde{\varrho}_{1}(k)|\hat{h}(k)|^{2} d k \leqq\left\|h_{1}\right\|^{2} \int_{\mathbb{R}^{3}} \tilde{\varrho}_{1}(k) d k=\|h\|_{1}^{2} \varrho_{c}<\infty .
$$

Let $W_{1}$ be the representation (2.4) with $A=f_{1}^{1 / 2}$ restricted to $L^{1} \cap L^{2}$ and put $W(h)=W_{1}(h) \otimes W_{0}(\hat{h}(0))$ and $\Omega=\Omega_{1} \otimes \Omega_{0}$. We have to check that $\Omega$ is cyclic for $W$. Since $\Omega_{1}$ is cyclic for $W_{1}$ and $\Omega_{0}$ is cyclic for $W_{0}$ it is enough to show that for all $h \in L^{1} \cap L^{2}, z \in \mathbb{C}$,

$$
W_{1}(h) \Omega_{1} \otimes W_{0}(z) \Omega_{0} \in V\left\{W(g) \Omega: g \in L^{1} \cap L^{2}\right\} .
$$

Now for all $h \in L^{1} \cap L^{2}$ and $z \in \mathbb{C}$ there exists a sequence $\left\{h_{n} \in L^{1} \cap L^{2}\right\}$ such that $\left\|h_{n}\right\| \rightarrow 0$ and

$$
\hat{h}_{n}(0) \rightarrow z-\hat{h}(0) \quad \text { as } \quad n \rightarrow \infty .
$$


Then

$$
W\left(h_{n}\right) W(h) \Omega=W_{1}\left(h_{n}+h\right) e^{-(i / 2) \operatorname{Im}\left\langle h_{n}, h\right\rangle} \Omega_{1} \otimes W_{0}\left(\hat{h}_{n}(0)+\hat{h}(0)\right) \Omega_{0}
$$

and

$$
\begin{aligned}
& \left\|W_{1}(h) \Omega_{1} \otimes W_{0}(z) \Omega_{0}-W\left(h_{n}\right) W(h) \Omega\right\|^{2} \\
& =2-2 \mu_{F}\left(h_{n}\right) \operatorname{Re}\left[\operatorname { e x p } \left\{-\frac{1}{2}\left\langle\hat{h}_{n}, \varrho_{1} \hat{h}_{n}\right\rangle-\frac{i}{2} \operatorname{Im}\left\langle h_{n}, h\right\rangle\right.\right. \\
& \left.\left.\quad-\frac{1}{2} \varrho_{0}\left|\hat{h}_{n}(0)+\hat{h}(0)-z\right|^{2}\right\}\right] \\
& \rightarrow 0 \text { as } n \rightarrow \infty .
\end{aligned}
$$

As in the case of the Araki-Woods representation, we can express this representation as a direct integral of factor representations

where

$$
W_{1}(h) \exp \left\{i 2^{1 / 2}\left(r-\varrho_{c}\right)^{1 / 2}(p \cos \theta+q \sin \theta)\right\},
$$

$$
\hat{h}(0)=p+i q,
$$

which are not gauge-invariant.

The generating functional $\mu_{\beta, \bar{\varrho}}$ is translation-invariant both above and below the critical density, but above critical it does not have the cluster property:

$$
\lim _{\|x\| \rightarrow \infty}\left|\mu_{\beta, \bar{\varrho}}\left(h_{x}+g\right) e^{-(i / 2) \operatorname{Im}\left\langle h_{x}, g\right\rangle}-\mu_{\beta, \bar{\varrho}}\left(h_{x}\right) \mu_{\beta, \bar{\varrho}}(g)\right| \neq 0
$$

for some $h, g \in L^{1} \cap L^{2}$, while below the critical density it has the property since the corresponding representation is a factor. When we consider time translations we see the advantage of taking $L^{1} \cap L^{2}$ as the space of test-functions. The space $\mathscr{D}$ is not invariant under the group of time translations corresponding to free-particle evolution $h \rightarrow h_{t}$ where $\hat{h}_{t}(k)=e^{-i t\|k\|^{2} / 2} \hat{h}(k)$, but $L^{1} \cap L^{2}$ is. Then $\mu_{\beta, \bar{\varrho}}$ on $L^{1} \cap L^{2}$ is time-translation invariant and has the KMS property.

\section{References}

1. Kac, M.: Private communication, 1971

2. Wieringa, J.D.: Groningen Thesis, Stellungen 3, Groningen: 1970

3. Araki, H., Woods, E. J.: J. Math. Phys. 4, 637 (1963)

4. Chaiken, J. H.: Commun. math. Phys. 8, 164 (1968)

5. Manuceau, J.: Ann. Inst. Henri Poincare 2, 139 (1968)

6. Manuceau, J., Verbeure, A.: Commun. math. Phys. 9, 293 (1968)

7. Penrose, O., Onsager, L.: Phys. Rev. 104, 576 (1956)

8. Arima, R.: J. Math. Kyoto Univ. 4, 207 (1964) 
9. Misohata, S., Arima, R.: J. Math. Kyoto Univ. 4, 245 (1964)

10. Hugenholtz, N.M.: Quantum mechanics of infinitely large systems, Proceedings of Noordwijk Summer Course. Amsterdam: North-Holland 1968

11. Pulè, J.: D. Phil. Thesis. Oxford: 1972

12. Araki, H.: J. Math. Phys. 1, 492 (1960)

13. Segal, I.E.: Canad. J. Math. 13, 1 (1961)

14. Cannon, J. T.: Commun. math. Phys. 29, 89 (1973)

Communicated by G. Gallavotti

J. T. Lewis

Dublin Institute for Advanced Studies

10 Burlington Road

Dublin 4, Ireland

J. V. Pulè

Mathematics Department

Royal University of Malta

Malta 